\title{
Integrated neural technologies: Solutions beyond tomorrow
}

Wael MY Mohamed ${ }^{1,2, *}$, Indranath Chatterjee ${ }^{3}$ and Mohammad A Kamal 4,5

${ }^{1}$ Clinical Pharmacology Department, Menoufia Medical School, Menoufia University, Egypt.

${ }^{2}$ Basic Medical Science Department, Kulliyyah of Medicine, International Islamic University (IIUM), Malaysia.

${ }^{3}$ Department of Computer Engineering, Tongmyong University, Busan, South Korea.

${ }^{4}$ King Fahd Medical Research Center, King Abdulaziz University, Saudi Arabia.

${ }^{5}$ Enzymoics, 7 Peterlee Place, Hebersham, NSW 2770; Novel Global Community Educational.

* Correspondence: wmy107@gmail.com; Tel.: +60-17-904-6810

Published: 24 October 2020

https://doi.org/10.31117/neuroscirn.v3i5.59

Keywords: neurotechnology; prosthetics; neuroimaging; brain-computer interface; nanotechnology

(C2020 by Mohamed et al for use and distribution in accord with the Creative Commons Attribution (CC BY-NC 4.0) license (https://creativecommons.org/licenses/by-nc/4.0/), which permits unrestricted non-commercial use, distribution, and reproduction in any medium, provided the original author and source are credited.

Neuroscience is an exciting area in which, at a fast rate, revolutionary advances materialise. Neurotechnology is interesting and contentious at the same time, as one of its aims is to "wire" human brains directly into computers. Neurotechnology is defined as the assembly of methods and instruments which allow a direct connection to the nervous system of technical components. These instruments are electrodes, machines or smart prostheses. They are designed to record and/or "translate" impulses from the brain into control instructions, or to modify brain function through the application of electrical or optical stimulation. The emergence of neuro-technologies is interdisciplinary. It supports the amalgamation of neurobiology with atomic, nano- and micro-sciences, as a fascinating path for significant development in the neuroscience domain. It poses a scientific foundation for potential therapeutic strategies.

Researchers may investigate brain function in people with diseases such as Alzheimer's disease, Schizophrenia, Parkinson's disease and epilepsy using technological devices, including MRI scanner and 3-D Computed Tomography. For instance, neurotechnological electrodes may be mounted on the surface of the head in the form of electrode caps, which is a "non-invasive" procedure. It is used, for example, in patients with amyotrophic lateral sclerosis (ALS) who are almost entirely paralysed during advanced stages of the disease. Furthermore, stimulating electrodes implanted in the brain are often used to externally excite or inhibit particular nucleus, regions or fibre bundles using an electrical current. The electrodes for "deep brain stimulation" (DBS), for example, are implanted with the utmost precision by a neurosurgeon in the respective regions deep within the brain. Any PD symptoms can be suppressed or enhanced by interference with these targets (Müller \& Rotter, 2017).

There are tons of exciting neural developments to keep track of, and several sources and experiences that will aid in the neurotechnology field. For instance, research is being undertaken to use technologies to offer people with prosthetics a sensory sensation, and neurostimulation also helped paralyse people move. Machines can aid the functioning of the human brain in this way. Brain-Machine Interface (BMI) or BrainComputer Interface $(\mathrm{BCl})$ can track the patient's psychological state towards therapy and behavioural modification. Recently, there are several attempts to Create electronic brain implants, or neuroprosthetics, which can convert the intention to step into the actual movement of a robotic system or a cursor on a computer screen. The goal is to improve the ability of paralysed patients to communicate with their surroundings and to bypass weakened spinal cords and return mobility to dead limbs. 
Alongside $\mathrm{BCl}$, neuroimaging also plays a significant role in the process of diagnosis and treatment of neurological and psychological disorders (Liotti \& Mayberg, 2001; Stephan, 2004). Neuroimaging is a branch to which several other fields contribute like computer science, neurology, physics, radiology, and et cetera. Several researchers across these disciplines make use of neuroimaging techniques to investigate the human brain. These techniques became very popular for the last 25 years, with the invention of functional magnetic resonance imaging (fMRI) (Chatterjee, 2018; Chatterjee et al., 2018). However, other neuroimaging techniques such as MRI (Chatteriee et al., 2020), Computed Tomography (CT), and Proton Emission Tomography (PET) (Nasrallah \& Dubroff, 2013) are used widely to identify the neurological problems in the brain. Few studies (Chatterjee et al., 2018; Liotti \& Mayberg, 2001) have already shown promising results with the usage of state-of-the-art neuroimaging techniques. The fMRI has a long history of identifying the neural biomarkers in the brain, be it for a psychological or neurological disorder. Even fMRI, MRI, and CT prove themselves as an effective tool for studying the healthy brain for different cognitive and neurological conditions.

Brain researches using neuroimaging techniques are increasing day by day with diverse study area, as in whole-brain analysis or region of interest (ROI) analysis, with the multidimensional problem statements, and interdisciplinary approaches. Modality coupling researches are getting attention to understand the brain functioning in schizophrenia (Chatterjee et al., 2019; Liotti \& Mayberg, 2001), epilepsy and Parkinson's diseases (Sabatini et al., 2000). Nowadays, electroencephalography (EEG)-MEGfMRI coupling is widely employed to study the direct (in case of EEG) and indirect (in case of fMRI) neuronal functionalities.
Psychological diseases such as schizophrenia need more studies to understand the underlying conditions. $\mathrm{BCl}$ and $\mathrm{fMRI}$ play a role in comprehending and supporting psychological diseases. As psychological disorders are challenging to diagnose with generic MRI or CT scan process, task-based and resting-state fMRI can help identify the condition in early stages and benefits in a better prognosis. The fMRI can detect the BOLD signal and thereby understanding the state of the neuronal activation at a particular region of the brain by measuring the oxygen inflow to that region (Chatterjee, 2018). It can thereby identify the probable chances and traces of neuron damage in different parts of the brain.

In this special issue of Neuroscience Research Notes journal, we are pleased to welcome original and unpublished articles on various topics related to technologies used in neuroscience. Also, we welcome papers that answer some of the ethical questions that occur in neurotechnology and discuss the interdisciplinary research on brain-machine interfaces. The submission may be in the form of comprehensive or extensive review, original research, short correspondence, report of the studies showing null or negative results, meta-analysis articles related to a wide variety of topics mentioned above in this article. We encourage the papers involving studies showing the result of such interdisciplinary integration or potential alliances. Computational approaches are commonly implemented in the discovery of scientific technology. Therefore, articles related to artificial intelligence and deep machine learning, particularly in brain changes concerning their structure and functions, will also be welcomed.

Conflicts of interest: The authors declare no conflict of interest.

\section{References}

Chatterjee, I. (2018). Mean deviation based identification of activated voxels from time-series fMRI data of schizophrenia patients. F1000Research, 7, 1615. https://doi.org/10.12688/f1000research.16405.2

Chatterjee, I., Agarwal, M., Rana, B., Lakhyani, N., \& Kumar, N. (2018). Bi-objective approach for computer-aided diagnosis of schizophrenia patients using fMRI data. Multimedia Tools and Applications, 77(20), 26991-27015. https://doi.org/10.1007/s11042-018-5901-0

Chatterjee, I., Kumar, V., Rana, B., Agarwal, M., \& Kumar, N. (2020). Identification of changes in grey matter volume using an evolutionary approach: an MRI study of schizophrenia. Multimedia Systems, 26(4), 383-396. https://doi.org/10.1007/s00530-020-00649-6

Chatterjee, I., Kumar, V., Sharma, S., Dhingra, D., Rana, B., Agarwal, M., \& Kumar, N. (2019). Identification of brain regions associated with working memory deficit in schizophrenia. F1000Research, 8, 124.

https://doi.org/10.12688/f1000research.17731.1

Liotti, M., \& Mayberg, H. S. (2001). The role of functional neuroimaging in the neuropsychology of depression. Journal of 
Clinical and Experimental Neuropsychology, 23(1), 121-136. https://doi.org/10.1076/jcen.23.1.121.1223

Müller, O., \& Rotter, S. (2017). Neurotechnology: Current Developments and Ethical Issues. Frontiers in Systems Neuroscience, 11, 93. https://doi.org/10.3389/fnsys.2017.00093

Nasrallah, I., \& Dubroff, J. (2013). An overview of PET neuroimaging. Seminars in Nuclear Medicine, 43(6), $449-461$. https://doi.org/10.1053/i.semnuclmed.2013.06.003

Sabatini, U., Boulanouar, K., Fabre, N., Martin, F., Carel, C., Colonnese, C., Bozzao, L., Berry, I., Montastruc, J. L., Chollet, F., \& Rascol, O. (2000). Cortical motor reorganisation in akinetic patients with Parkinson's disease. A functional MRI study. Brain, 123(2), 394-403. https://doi.org/10.1093/brain/123.2.394

Stephan, K. E. (2004). On the role of general system theory for functional neuroimaging. Journal of Anatomy, 205(6), 443470. https://doi.org/10.1111/i.0021-8782.2004.00359.x 